\title{
CÉRIO: PROPRIEDADES CATALÍTICAS, APLICAÇÕES TECNOLÓGICAS E AMBIENTAIS
}

\section{Tereza S. Martins*}

Instituto de Física, Universidade de São Paulo, CP 66318, 05315-970 São Paulo - SP, Brasil

Thiago L. R. Hewer

Instituto de Química, Universidade de São Paulo, CP 26077, 05513-970 São Paulo - SP, Brasil

Renato S. Freire

Instituto de Química, Universidade de São Paulo, CP 26077, 05513-970 São Paulo - SP/ Centro de Capacitação e Pesquisa em Meio Ambiente, Universidade de São Paulo, Cubatão - SP, Brasil

Recebido em 13/11/06; aceito em 2/3/07; publicado na web em 25/10/07

\begin{abstract}
CERIUM: CATALYTIC PROPERTIES, TECHNOLOGICAL AND ENVIRONMENTAL APPLICATIONS. Cerium based-compounds have great importance in a wide range of technological applications, such as: fuel cell devices development; metallurgic processes, petroleum refining; glass and ceramic production. Recently, its catalytic properties have been also explored for environmental applications, especially those to prevent or to control atmospheric and water pollution. Subjects covered in this work include a brief description of the fundaments of cerium catalytic properties and some relevant technological applications. Special attention is given to its photocatalytic activity and its ability to degrade pollutants. Recent results and future prospect about these applications are also evaluated.
\end{abstract}

Keywords: lanthanide; catalysis; heterogeneous photocatalyst.

\section{INTRODUÇÃO}

O cério, elemento químico de número atômico 58 , é um dos 15 elementos classificados como lantanídeos ( $\mathrm{La}$ ao $\mathrm{Lu}$ ), que juntamente com Sc e Y formam a família das Terras Raras. Cério é o mais abundante das Terras Raras. Sua abundância na crosta terrestre é da ordem de $60 \mathrm{ppm}$, o que o torna o $26^{\circ}$ elemento em frequiência de ocorrência, sendo quase tão abundante quanto cobre e níquel. Apesar de o cério ser o lantanídeo de maior ocorrência, é encontrado em muitos minerais somente em níveis traço, sendo a bastinasita e monasita os minerais mais importantes como fonte de cério e suprem a maior parte da demanda mundial deste elemento ${ }^{1-6}$. Estes dois minerais, dependo da localidade dos depósitos, possuem cerca de 65$70 \%$ (bastinasita) e 49-74\% (monasita) de terras céricas (La-Gd), sendo que, considerando-se a porcentagem de lantanídeos na bastinasita e monasita como $100 \%$, a quantidade de $\mathrm{CeO}_{2}$ corresponde a aproximadamente 49 e $47 \%$, respectivamente ${ }^{5}$.

$\mathrm{O}$ cério, que tem a configuração eletrônica $[\mathrm{Xe}] 4 \mathrm{f}^{1} 5 \mathrm{~d}^{1} \mathrm{~s}^{2}$, é muito eletropositivo e suas interações são predominantemente iônicas devido ao baixo potencial de ionização $\left(3,49 \mathrm{~kJ} \mathrm{~mol}^{-1}\right)$ para a remoção dos três primeiros elétrons ${ }^{1-3}$. Assim como outros lantanídeos, o estado de oxidação mais estável deste elemento é o (+III). O cério trivalente tem propriedades semelhantes a outros íons lantanídeos com mesmo estado de oxidação ${ }^{3,5}$, com exceção da sua fácil oxidação para $\mathrm{Ce}^{4+}$ e instabilidade em ar e água. Seus sais, em geral, mais estáveis que os de $\mathrm{Ce}^{4+}$, são pouco hidrolisáveis e muito utilizados como precursores para vários compostos de cério ${ }^{1-6}$. Diferentemente dos demais elementos dessa classe, o estado de oxidação (+IV) também é estável, principalmente em virtude da configuração eletrônica semelhante à de um gás nobre $\left([\mathrm{Xe}] 4 \mathrm{f}^{0}\right)$. Este elemento é o único lantanídeo, no estado tetravalente, estável em solução aquosa, porém a influência da carga maior e tamanho iônico menor faz com que os sais do íon $\mathrm{Ce}^{4+}$ sejam hidrolisados

*e-mail: tmartins@if.usp.br em soluções aquosas com mais facilidade que o íon $\mathrm{Ce}^{3+}$ e, em conseqüência disso, estas soluções são fortemente ácidas ${ }^{5}$. No estado tetravalente, o cério é um agente oxidante forte e pode ser reduzido, por exemplo, por ácidos orgânicos ${ }^{5}$, sais ferrosos, peróxido de hidrogênio e outros compostos inorgânicos e orgânicos, uma vez que o potencial padrão de redução $\left(\mathrm{E}^{0}\right)$ para o par $\mathrm{Ce}(\mathrm{IV}) / \mathrm{Ce}(\mathrm{III})$ é de aproximadamente $1,6 \mathrm{~V}$ (com algumas variações dependendo do ânion e do meio $)^{5}$. Apesar deste potencial, soluções aquosas contendo Ce(IV) são estáveis, provavelmente por efeitos cinéticos. Em virtude dessas propriedades de óxido-redução, o cério é muito conhecido e utilizado em uma série de reações de síntese orgâni$\mathrm{ca}^{5,7}$ e de métodos analíticos ${ }^{5}$.

Dentre os compostos de $\mathrm{Ce}^{4+}$, o dióxido de cério $\left(\mathrm{CeO}_{2}\right.$, também chamado de céria) é o mais estável em virtude de sua estrutura cúbica tipo fluorita (grupo espacial $F m 3 m)^{3,5}$. Tal estrutura confere a este óxido uma estabilidade maior que o sesquióxido, $\mathrm{Ce}_{2} \mathrm{O}_{3}$, com cério trivalente (estrutura hexagonal) ${ }^{3}$. $\mathrm{O} \mathrm{CeO}_{2}$ pode ser obtido a partir de sais precursores por calcinação em ar ou meio que contém oxigênio; o óxido de cério não estequiométrico, $\mathrm{CeO}_{2-\mathrm{x}}(\mathrm{x}$ pode ser acima de 0,3$)$, também pode ser obtido e neste caso, utilizam-se dopantes, tais como óxidos de outras terras raras, como por exemplo, Y ou La. A introdução desses elementos na rede cristalina do dióxido de cério faz com que ocorra uma compensação de cargas, gerando vacâncias de oxigênio, sendo que a mobilidade do oxigênio depende do tipo de dopante empregado ${ }^{1-6}$.

Os compostos de cério têm um enorme potencial para aplicação em diversas vertentes tecnológicas, principalmente devido a algumas de suas propriedades, tais como faixa de potencial redox, alta mobilidade de oxigênio na rede cristalina, alta afinidade por compostos contendo oxigênio, nitrogênio e enxofre ${ }^{1-14}$. Algumas dessas aplicações são mostradas no fluxograma da Figura 1.

Uma das principais aplicações de compostos à base de cério é em processos metalúrgicos, onde estes compostos são adicionados ao aço para eliminar impurezas, principalmente oxigênio e enxofre, devido à sua alta afinidade para formar ligações com esses 


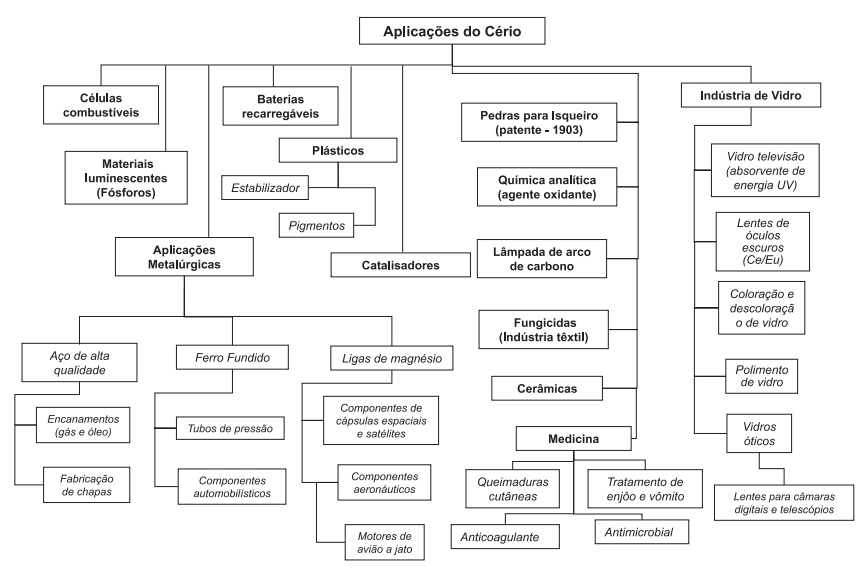

Figura 1. Fluxograma de algumas aplicações dos compostos de cério

elementos, contribuindo, assim, para melhorar significativamente a qualidade do aço, aumentando, por exemplo, a resistência e ductibilidade $^{4-14}$. Com esta mesma finalidade, estes compostos também podem ser adicionados a outras ligas metálicas, tais como ligas à base de cobalto ou níquel, que são utilizadas, por ex., em turbinas de motores de aviões ${ }^{8,9}$. O cério, na forma de sais, cloretos e nitratos, também é utilizado para evitar corrosão de utensílios de alumínio (e suas ligas), substituindo os íons cromatos, que apresentam implicações negativas do ponto de vista ambiental ${ }^{5-14}$.

Uma outra aplicação relevante do cério é em células a combustíveis. Estas células têm grande importância na atualidade, principalmente na busca de alternativas para a geração de energia elétrica de forma limpa e eficiente. Um esquema geral do mecanismo de funcionamento de uma célula a combustível é apresentado na Figura 2. As células com eletrólito de óxido sólido baseiam-se em um eletrólito condutor de íons oxigênio, por meio do qual os íons $\mathrm{O}^{2-}$ migram do cátodo até o ânodo, onde reagem com um combustível, gerando uma corrente elétrica no sistema. No caso do cério, esta condução iônica se dá em virtude dos efeitos das vacâncias de oxigênio existentes na estrutura cristalina do $\mathrm{CeO}_{2}$. Existem vários outros tipos de condutores de íons $\mathrm{O}^{2-}$, os mais tradicionais são à base de zircônia, porém os eletrólitos de $\mathrm{CeO}_{2}$ têm sido preferidos, devido a sua alta condutividade iônica, boa compatibilidade com outros materiais constituintes das células e capacidade de operar em uma faixa de temperatura menor (entre 500 e $700{ }^{\circ} \mathrm{C}$, contra cerca de $1000{ }^{\circ} \mathrm{C}$ necessários quando se utilizam eletrólitos tradicionais) $)^{15-20}$.

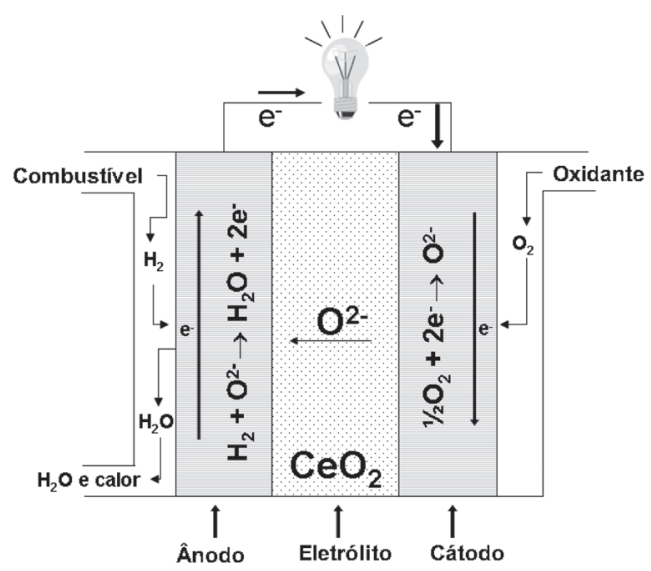

Figura 2. Representação esquemática do funcionamento de uma célula a combustível: Ânodo: ocorre a reação de oxidação e liberação de elétrons que percorrem o circuito externo, produzindo energia elétrica por meio de uma carga externa; cátodo: ocorre a reação de redução

\section{CATÁLISE}

As propriedades catalíticas dos compostos de cério têm sido exploradas em uma ampla gama de aplicações ${ }^{4-9,21-46}$. Neste trabalho dedica-se especial atenção ao emprego destas propriedades em sistemas que podem minimizar efeitos deletérios de uma série de processos antrópicos sobre o meio ambiente.

Uma destas aplicações é o uso de compostos à base de cério em sistemas para depuração de emissões gasosas automotivas (comumente conhecidos como "catalisadores"). Um dos maiores problemas ambientais nas grandes cidades é a poluição do ar, sendo que a queima de combustíveis fósseis durante as atividades de transporte é uma das principais causas desta poluição ${ }^{29,33,39}$. Idealmente, em um motor de combustão interna, a queima do combustível deveria ser completa produzindo somente água, dióxido de carbono e nitrogênio como os principais gases de escape. No entanto, devido à mistura não estequiométrica dos reagentes (combustível e $\mathrm{O}_{2}$ ), têm-se a liberação de hidrocarbonetos, monóxido de carbono, óxidos de nitrogênio e outras substâncias (por ex., óxidos de enxofre). A emissão destes gases pode alterar a concentração e distribuição (equilíbrio) de uma série de espécies químicas na atmosfera, tais como $\mathrm{NO}_{\mathrm{x}}$ que participam de reações fotoquímicas/radicalares na troposfera levando à geração de espécies altamente oxidantes, como o ozônio; composição e estruturação de materiais particulados; alteração do $\mathrm{pH}$ da água da chuva etc. Tais espécies na atmosfera trazem grandes implicações à saúde pública e qualidade de vida da população dos grandes centros urbanos $22,29,33$. Estima-se que aproximadamente 50 milhões de carros são produzidos anualmente e que mais de 700 milhões estão em utilização no mundo inteiro ${ }^{29}$; tais números mostram que o uso de catalisadores para purificar os gases de escape é absolutamente necessário e indispensável.

Os catalisadores mais empregados atualmente para essa finalidade são os conhecidos como catalisadores tipo três vias (CTV). Este tipo de catalisador é constituído por vários componentes, dentre os quais se destacam metais nobres (exemplo: Pt, Pd e Rh) que atuam como sítio ativo durante as reações de redução; alumina que devido a sua alta área superficial atua como um excelente suporte; óxidos metálicos (ex.: óxido de cério, lantânio, níquel, zircônio e bário) que têm papel de promotores ou estabilizadores ${ }^{35}$. Duas das principais funções dos catalisadores CTV são oxidar hidrocarbonetos e $\mathrm{CO}$ a $\mathrm{CO}_{2}$ e reduzir os $\mathrm{NO}_{x}$ a $\mathrm{N}_{2}$. Nestes processos, o $\mathrm{CeO}_{2}$ atua como armazenador de oxigênio e como estabilizador térmico/estrutural, conforme Equações 1 a $3^{22}$ :

$$
\begin{aligned}
& \mathrm{CeO}_{2}+\mathrm{xCO} \rightarrow \mathrm{CeO}_{2-\mathrm{x}}+\mathrm{xCO}_{2} \\
& \mathrm{CeO}_{2}+\mathrm{C}_{\mathrm{x}} \mathrm{H}_{\mathrm{y}} \rightarrow \mathrm{CeO}_{2-(2 \mathrm{x}+0,5 \mathrm{y})}+\mathrm{xCO}_{2}+0,5 \mathrm{yH}_{2} \mathrm{O} \\
& \mathrm{CeO}_{2-\mathrm{x}}+\mathrm{xNO} \rightarrow \mathrm{CeO}_{2}+0,5 \mathrm{xN}_{2}
\end{aligned}
$$

Essas reações acontecem simultaneamente, sendo necessário manter a relação entre os poluentes e o oxigênio próxima ao ponto estequiométrico. Assim, o $\mathrm{CeO}_{2}$ tem a função de fornecer oxigênio de sua própria rede cristalina quando a mistura está com falta de oxigênio; por outro lado, quando há excesso de oxigênio esta superfície é reoxidada (Equações 4 e 5). Além dessa função reguladora de oxigênio, a presença de $\mathrm{CeO}_{2}$ também evita que os catalisadores CTV percam a eficiência em altas temperaturas, pois ela inibe/ desloca as transições de fase de outros materiais como, por ex., do suporte de alumina ${ }^{16,19,22}$.

$$
\begin{aligned}
& \mathrm{CeO}_{2} \rightarrow \mathrm{CeO}_{2-\mathrm{x}}+0,5 \mathrm{xO}_{2} \\
& \mathrm{CeO}_{2-\mathrm{x}}+0,5 \mathrm{xO}_{2} \rightarrow \mathrm{CeO}_{2}
\end{aligned}
$$


A adição de íons lantânio, zircônia, $\mathrm{BaO}$ e $\mathrm{SiO}_{2}$ à estrutura do $\mathrm{CeO}_{2}$ pode contribuir para aumentar a quantidade de defeitos na rede cristalina e proporcionar ao $\mathrm{CeO}_{2}$ maior capacidade para armazenar oxigênio ${ }^{22,27-33,35,38,42-45}$, melhorando o desempenho dos catalisadores CTV. Atualmente, o uso de $\mathrm{CeO}_{2}$ neste tipo de catalisador é uma das principais aplicações tecnológicas deste elemento e tem consumido a maior parte do cério produzido mundialmente ${ }^{4,6}$.

Uma outra aplicação catalítica do cério com relação a emissões gasosas é nas unidades de Craqueamento Catalítico Fluido $(\mathrm{CCF})$, onde compostos à base de cério têm sido utilizados para a remoção de $\mathrm{SO}_{x}$ nesta etapa de refino de petróleo ${ }^{36}$. O cério promove a oxidação do $\mathrm{SO}_{2}$ a $\mathrm{SO}_{3}$, sendo que seus sítios básicos permitem a adsorção de $\mathrm{SO}_{2} / \mathrm{SO}_{3}$ com formação de sulfatos, tornando muito mais fácil a dessorção redutiva de sulfatos a sulfeto de hidrogênio $\left(\mathrm{H}_{2} \mathrm{~S}\right)$ sob condições redutivas. Um possível esquema para estas reações é apresentado nas Equações 6 e 7

$\mathrm{CeO}_{2}+2 \mathrm{SO}_{3} \leftrightarrow \mathrm{Ce}\left(\mathrm{SO}_{4}\right)_{2}$

$\mathrm{Ce}\left(\mathrm{SO}_{4}\right)_{2}+8 \mathrm{H}_{2} \leftrightarrow \mathrm{CeO}_{2}+2 \mathrm{H}_{2} \mathrm{~S}+6 \mathrm{H}_{2} \mathrm{O}$

$\mathrm{O} \mathrm{CeO}_{2}$ pode contribuir em todas as etapas citadas, pois seu potencial padrão de redução facilita a oxidação de $\mathrm{SO}_{2}$ para $\mathrm{SO}_{3}$ sob as condições de regeneração do CCF. Como produto desta reação com $\mathrm{SO}_{2}$ tem-se o óxido de cério subestequiométrico, o qual é reoxidado pelo oxigênio (Equações 8 e 9) 21,32,36 $^{2}$

$\mathrm{xSO}_{2}+\mathrm{CeO}_{2} \leftrightarrow \mathrm{xSO}_{3}+\mathrm{CeO}_{2-\mathrm{x}}$

$\mathrm{CeO}_{2-\mathrm{x}}+0,5 \mathrm{xO}_{2} \leftrightarrow \mathrm{CeO}_{2}$

Catalisadores à base de cério também são utilizados em técnicas de oxidação úmida catalítica (OUC) no tratamento de compostos orgânicos voláteis (COV) provenientes de uma gama de processos produtivos industriais. Os COV são considerados poluentes tóxicos com potencial mutagênico, carcinogênico e teratogênico. Diferentes tecnologias têm sido desenvolvidas para a destruição de $\mathrm{COV}$, sendo a oxidação úmida catalítica uma das mais versáteis e eficientes, pois consegue remover quantitativamente uma ampla variedade de $\mathrm{COV}$. No processo COV o principal papel do $\mathrm{CeO}_{2}$ é fornecer, transportar e ativar o oxigênio durante o processo de oxidação. Para essa finalidade, geralmente emprega-se $\mathrm{CeO}_{2}$ dopado com metais de transição, pois busca-se aumentar a estabilidade térmica e a área superficial deste material, visando aumentar a transferência eletrônica durante os processos redox e a taxa de difusão do oxigênio ${ }^{23,24,27,39}$.

Via este processo de oxidação, o óxido de cério tem sido utilizado, por exemplo, para degradação de ácido acrílico $\left(\mathrm{C}_{3} \mathrm{H}_{4} \mathrm{O}_{2}\right)$, composto amplamente usado em diversas indústrias e com grande potencial tóxico, o qual quando lançado sem tratamento em efluentes industriais pode causar sérios danos ao meio ambiente, principalmente nos sistemas aquáticos ${ }^{39}$. Esse ácido é um composto refratário, sendo muito estável termicamente (suporta temperaturas de até $300{ }^{\circ} \mathrm{C}$ ). Silva e colaboradores ${ }^{39}$ estudaram a degradação deste composto empregando diferentes materiais à base de $\mathrm{CeO}_{2}$. Os melhores resultados foram obtidos com uma mistura de $\mathrm{Ru} / \mathrm{CeO}_{2}$. Estes estudos também mostraram que catalisadores à base de $\mathrm{Mn} /$ $\mathrm{CeO}_{2}$ apresentam alta seletividade para oxidação do ácido acrílico, promovendo uma redução de $94,1 \%$ no teor de carbono orgânico total (COT) em somente $30 \mathrm{~min}$ de tratamento ${ }^{39}$.

$\mathrm{O}$ processo de $\mathrm{OUC}$ via $\mathrm{CeO}_{2}$ também mostrou-se muito eficiente tanto para a diminuição na concentração de fenol quanto para a completa mineralização (oxidação em $\mathrm{CO}_{2}$ e $\mathrm{H}_{2} \mathrm{O}$ ) da matéria orgânica deste composto ${ }^{46}$. Lin e colaboradores ${ }^{46}$ realizaram um estudo empregando diferentes concentrações iniciais de fenol (variando desde 400 até $5000 \mathrm{mg} \mathrm{L}^{-1}$ ). Observaram que a mineralização da matéria orgânica foi superior a $90 \%$ para todas as concentrações de fenol, exceto para a concentração de $5000 \mathrm{mg} \mathrm{L}^{-1}$, para a qual foi obtida uma máxima redução de $75 \%$. Estes valores de degradação e mineralização foram obtidos empregando-se entre 1 e $2 \mathrm{~g}$ de $\mathrm{CeO}_{2}$ para cada litro de solução de fenol; pressão parcial de $\mathrm{O}_{2}$ de 1 a 1,5 $\mathrm{MPa}$ e temperatura de aproximadamente $180{ }^{\circ} \mathrm{C}$. As variações nas condições reacionais foram realizadas em função do mecanismo de atuação do catalisador no processo de OUC, onde a capacidade do cério em atuar como agente oxidante e redutor (balanço estequiométrico de oxigênio na matriz do $\mathrm{CeO}_{2}$ ) é dependente da concentração do $\mathrm{O}_{2}$ no meio reacional. $\mathrm{O}$ modelo de mecanismo para as reações de oxidação em OUC $^{46}$ é apresentado na Figura 3.

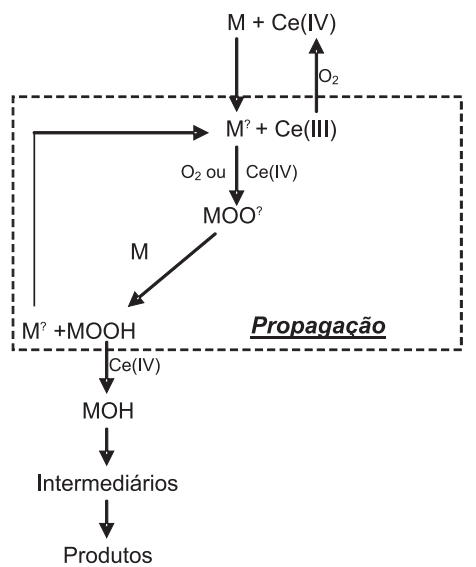

Figura 3. Representação do mecanismo de atuação do catalisador de cério na degradação de compostos orgânicos poluentes por OUC. $M=$ compostos orgânicos

A influência do cério no processo OUC também foi evidenciada no trabalho de Abecassis-Wolfovich e colaboradores ${ }^{47}$, onde foi estudada a incorporação de íons de cério em matriz de óxido de manganês do tipo criptomelano (OMS-2), a qual é uma estrutura porosa ordenada. A incorporação dos íons de cério no OSM-2 aumentou a capacidade de degradação de fenol por um fator de 1,5 e 3 vezes quando comparado com um catalisador de óxido de manganêscério desordenado e carvão ativado, respectivamente.

Oliveiro e colaboradores ${ }^{48}$ também avaliaram a eficiência de catalisadores à base de cério pelo processo OUC na degradação de compostos recalcitrantes. Estudaram a oxidação do ácido maleico por $\mathrm{CeO}_{2} / \mathrm{Ru}$ e observaram um aumento na degradação deste ácido de cerca de $40 \%$ e uma mineralização 4,5 vezes maior quando na presença do catalisador ${ }^{46-51}$.

\section{FOTOCATÁLISE HETEROGÊNEA}

Apesar de ser eficiente na degradação de compostos orgânicos, o processo OUC requer condições relativamente drásticas (principalmente com relação à temperatura e pressão parcial de oxigênio). Assim, a fotocatálise heterogênea apresenta-se como uma alternativa que permite a degradação de uma série de compostos orgânicos poluentes sob condições mais brandas, sendo que compostos à base de cério também podem ser empregados como fotocatalisadores neste processo ${ }^{52,53}$.

O mecanismo de degradação de poluentes via processo de fotocatálise heterogênea baseia-se na geração de espécies radicalares, as quais irão promover a oxidação dos compostos-alvo. Para esta 
geração de radicais, primeiramente necessita-se excitar eletronicamente o material catalisador, visando gerar um par lacuna/elétron $\left(h^{+} / e^{-}\right)$. A formação deste par ocorre pela incidência de radiação nas partículas do semicondutor, produzindo a excitação de um elétron da banda de valência para a banda de condução, tendo assim, a geração de sítios oxidativos na banda de valência $\left(\mathrm{h}^{+}{ }_{\text {bv }}\right)$ e sítios redutivos na banda de condução $\left(\mathrm{e}_{\mathrm{bc}}^{-}\right.$). Com a migração do par $\mathrm{h}^{+} / \mathrm{e}^{-}$ para a superfície da partícula, os sítios redox podem interagir com moléculas adsorvidas em sua superfície e mediar reações que podem levar à degradação das espécies poluentes ${ }^{52-55}$.

Esta degradação dos compostos orgânicos poluentes pode ocorrer por dois mecanismos distintos: direto e indireto. $\mathrm{O}$ mecanismo direto envolve a oxidação ou redução das moléculas dos poluentes na superfície do semicondutor pelas lacunas $\left(\mathrm{h}^{+}{ }_{\mathrm{bv}}\right)$ e pelos elétrons fotogerados (Equações 10 e 11) ) $^{52-55}$

$$
\begin{aligned}
& \mathrm{M}^{\mathrm{n}}+\mathrm{h}^{+}{ }_{\mathrm{BV}} \rightarrow \mathrm{M}^{\mathrm{n}+1} \\
& \mathrm{M}^{\mathrm{n}}+\mathrm{e}_{\mathrm{BC}} \rightarrow \mathrm{M}^{\mathrm{n}-1}
\end{aligned}
$$

Os elementos da série dos lantanídeos apresentam facilidade para a complexação com bases de Lewis ${ }^{56-58}$. Desta forma, a dopagem de fotocatalisadores com o cério aumenta a capacidade de adsorção dos poluentes, (tais como ácidos orgânicos, aldeídos, aminas e álcoois) sobre a superfície do fotocatalisador, o que pode favorecer o processo de fotodegradação dos poluentes ${ }^{57,58}$.

Xie e colaboradores ${ }^{58}$ realizaram um estudo da influência dos íons lantanídeos na dopagem do $\mathrm{TiO}_{2}$ e seu efeito no desempenho catalítico sobre a degradação do azo corante X-3B. Os íons $\mathrm{Ce}^{4+}$, $\mathrm{Nd}^{3+}$ e $\mathrm{Eu}^{3+}$ foram empregados na dopagem do catalisador de $\mathrm{TiO}_{2}$ sintetizado por co-precipitação. As capacidades de adsorção dos fotocatalisadores dopados com íons lantanídeos foram superiores ao $\mathrm{TiO}_{2}$ puro. $\mathrm{O} \mathrm{Ce}^{4+}-\mathrm{TiO}_{2}$ apresentou uma saturação máxima de $0,0413 \mathrm{mmol}$ de corante por grama de catalisador, enquanto que o $\mathrm{TiO}_{2}$ teve uma saturação máxima de $0,0345 \mathrm{mmol} \mathrm{g}^{-1}$, já o $\mathrm{Nd}^{3+}$ $\mathrm{TiO}_{2}$ e o $\mathrm{Eu}^{3+}-\mathrm{TiO}_{2}$ obtiveram saturação de 0,0500 e $0,0579 \mathrm{mmol} \mathrm{g}$ ${ }^{1}$, respectivamente. Efetivamente a incorporação dos íons lantanídeos proporcionou um aumento na capacidade de adsorção na superfície das partículas dos catalisadores, através da interação dos lantanídeos com o grupo $\mathrm{N}=\mathrm{N}$ do azocorante. A maior capacidade de adsorção dos catalisadores dopados com os lantanídeos refletiu em um aumento na eficiência de degradação do processo de fotocatálise heterogênea. As cinéticas de pseudo-primeira ordem do processo de degradação para os diferentes fotocatalisadores apresentaram a seguinte ordem decrescente: $\mathrm{TiO}_{2}<\mathrm{Ce}^{4+}-\mathrm{TiO}_{2}<\mathrm{Eu}^{3+}-\mathrm{TiO}_{2}<\mathrm{Nd}^{3+}-$ $\mathrm{TiO}_{2}$. A degradação para um período de $120 \mathrm{~min}$ de tratamento do azocorante foi de $89,1 \%$ empregando-se $\mathrm{Nd}^{3+}-\mathrm{TiO}_{2}, 85,3 \%$ para $\mathrm{Eu}^{3+}-$ $\mathrm{TiO}_{2}$ e $80,2 \%$ para $\mathrm{Ce}^{4+}-\mathrm{TiO}_{2}$. Quando se empregou $\mathrm{TiO}_{2}$ puro nas mesmas condições reacionais, obteve-se uma degradação máxima do corante de $67,9 \%$.

Além do mecanismo direto, a oxidação dos compostos orgânicos poluentes ocorre através da geração de radicais hidroxila, mecanismo indireto. Assim, a degradação dos poluentes dá-se pela oxidação dos compostos orgânicos pelos radicais hidroxilas fotogerados na superfície do semicondutor. Devido ao elevado potencial de redução $\left(\mathrm{E}^{0}=2,7 \mathrm{~V}\right)$; alta reatividade e baixa seletividade dos radicais hidroxilas, esta rota costuma ser mais eficiente na remoção de compostos orgânicos poluentes ${ }^{53-55}$. Sucessivas interações da matéria orgânica com os radicais hidroxilas, durante as reações em cadeia, podem levar à completa mineralização destes compostos (Equação 12)

$\cdot \mathrm{OH}+\mathrm{M}^{\mathrm{n}} \rightarrow \mathrm{M}^{\mathrm{n}-1} \stackrel{\cdot \mathrm{OH} \approx}{\longrightarrow} \mathrm{CO}_{2}+\mathrm{H}_{2} \mathrm{O}$
As possíveis reações envolvidas na geração dos radicais não são completamente compreendidas, entretanto, a geração dos radicais pode ser exemplificada pelas Equações 13 a 20, de acordo com cada mecanismo específico ${ }^{55}$

$$
\begin{aligned}
& \mathrm{SC}+(\mathrm{hv} \geq \mathrm{Eg}) \rightarrow \mathrm{H}^{+}{ }_{\mathrm{BV}}+\mathrm{e}_{\mathrm{BC}}^{-} \\
& \mathrm{h}^{+}+\mathrm{H}_{2} \mathrm{O} \rightarrow \cdot \mathrm{OH}+\mathrm{H}^{+} \\
& \mathrm{OH}^{-}+\mathrm{h}^{+} \rightarrow \cdot \mathrm{OH} \\
& \mathrm{O}_{2}+\mathrm{e}^{-} \rightarrow \mathrm{O}_{2}^{-} \\
& \mathrm{O}_{2}^{-}+\mathrm{H}^{+} \rightarrow \mathrm{HO}_{2}^{\bullet} \\
& \mathrm{HO}_{2} \cdot+\mathrm{HO}_{2}^{\bullet} \rightarrow \mathrm{H}_{2} \mathrm{O}_{2}+\mathrm{O}_{2} \\
& \mathrm{H}_{2} \mathrm{O}_{2} \cdot+\mathrm{e}^{-} \rightarrow \mathrm{OH}^{-}+\bullet \mathrm{OH} \\
& \mathrm{H}_{2} \mathrm{O}_{2}+\mathrm{O}_{2}^{-} \cdot \rightarrow \mathrm{OH}^{-}+\cdot \mathrm{OH}+\mathrm{O}_{2}
\end{aligned}
$$

Sem a presença dos fotocatalisadores a geração dos radicais hidroxilas a partir da água, via reações fotoquímicas, necessita de radiação com comprimentos de onda menores que $200 \mathrm{~nm}$. Desta forma, os catalisadores são de fundamental importância para a geração dos radicais de uma forma mais aplicável, ou seja, com o uso de radiações menos energéticas ${ }^{52-55}$. Assim, a eficiência do processo de fotocatálise heterogênea está relacionada com a capacidade do semicondutor em atuar como fotocatalisador na geração de radicais. Dentre os semicondutores conhecidos, o $\mathrm{TiO}_{2}$ é o semicondutor mais vastamente empregado no processo de fotocatálise ${ }^{59,60}$. $\mathrm{O} \mathrm{TiO}_{2}$ é um semicondutor do tipo-n e sua energia de "band-gap" é da ordem de 3,2 eV, dependendo da fase cristalina predominante, anatase ou rutilo, sendo que sua excitação pode ser obtida empregando-se radiações com $\lambda \leq 385 \mathrm{~nm}^{54,60}$.

$\mathrm{O} \mathrm{CeO}_{2}$, assim como o $\mathrm{TiO}_{2}$, também é um semicondutor, com uma energia de "band-gap" é de 2,94 eV, a qual possibilita a sua ativação fotônica em comprimentos de ondas menos energéticos, próximos à região do espectro visível. Economicamente, pode-se ter um menor gasto energético já que há um melhor aproveitamento da radiação proveniente do sol. Do ponto de vista tecnológico a confecção de reatores sem a necessidade de utilização de lâmpadas de luz ultravioleta e materiais transparentes a esta radiação pode facilitar a aplicação do processo de fotocatálise heterogênea na degradação de compostos poluentes ${ }^{61}$.

Estas características mostram que a aplicação do $\mathrm{CeO}_{2}$ para degradação de compostos poluentes pode ser vantajosa para aumentar a aplicabilidade do processo de fotocatálise heterogênea. No entanto, a utilização do $\mathrm{CeO}_{2}$ como fotocatalisador e sua capacidade de gerar espécies radicalares não é muito explorada, sendo demonstrada, até o presente momento, em poucos trabalhos científicos ${ }^{61,62}$.

Hernández-Alonso e colaboradores ${ }^{62}$ mostraram, através de estudos de EPR, a geração de radicais superóxido $\left(\mathrm{O}_{2}{ }^{-}\right)$na superfície do $\mathrm{CeO}_{2}$ via ativação fotônica deste semicondutor. A atividade fotocatalítica dos semicondutores foi avaliada na degradação de tolueno. Comparando-se as velocidades de degradação do tolueno pelo $\mathrm{CeO}_{2}$ com o $\mathrm{TiO}_{2}$, observou-se que este último semicondutor apresentou velocidades, para a degradação do tolueno, com uma ordem de grandeza superior ao obtido com $\mathrm{CeO}_{2}\left(\mathrm{k}_{\mathrm{TiO} 2}=6,0 \times 10^{-10}\right.$ mol s${ }^{-1} \mathrm{~m}^{-2} ; \mathrm{k}_{\mathrm{CeO} 2}=1,2 \times 10^{-11} \mathrm{~mol} \mathrm{~s}^{-1} \mathrm{~m}^{-2}$ ). Entretanto, o óxido de cério (IV) permitiu uma mineralização total do tolueno, enquanto que os catalisadores à base de titânio não levaram a uma completa oxidação da matéria orgânica deste poluente. Um outro estudo re- 
alizado por Alonso e colaboradores ${ }^{62}$ comprovou a fotogeração dos radicais $\mathrm{OH}$ pelo $\mathrm{CeO}_{2}$. Estes autores também testaram a atividade catalítica do $\mathrm{CeO}_{2}$ na degradação de tolueno, obtendo resultados melhores que os descritos anteriormente (velocidade de reação de degradação do poluente foi de $8,7 \times 10^{-11} \mathrm{~mol} \mathrm{~s}^{-1} \mathrm{~m}^{-2}$, com uma conversão total da matéria orgânica em $\mathrm{CO}_{2}$ e $\mathrm{H}_{2} \mathrm{O}$ ).

Apesar de promover a mineralização dos poluentes, as constantes cinéticas obtidas com o emprego de $\mathrm{CeO}_{2}$ em processos fotocatalíticos são relativamente baixas. Assim, algumas estratégias de modificação destes semicondutores estão sendo estudadas para melhorar seu desempenho fotocatalítico. As principais abordagens neste sentido são: deposição de metais nobres e metais de transição na superfície das partículas dos semicondutores; dopagem dos catalisadores com metais de transição e emprego de semicondutores de óxidos mistos ${ }^{63}$.

Estas duas últimas opções têm demonstrado um grande potencial de aplicação, principalmente por permitir a utilização e incremento das propriedades catalíticas de materiais já consagrados, como o $\mathrm{TiO}_{2}$. Alguns trabalhos relatam o emprego do $\mathrm{CeO}_{2}$ na dopagem do $\mathrm{TiO}_{2}$, demonstrando a capacidade deste material em aumentar o desempenho catalítico do $\mathrm{TiO}_{2}$. Xu e colaboradores ${ }^{64}$ estudaram a dopagem do $\mathrm{TiO}_{2}$ com cério, nas seguintes razões estequiométricas: 0,$05 ; 0,1 ; 0,2 ; 0,5$ e $1 \%$ de Ce. Relataram que a presença de cério proporcionou um deslocamento da energia de "band-gap" do $\mathrm{TiO}_{2}$ para comprimentos de ondas maiores, deslocando-o da região do UV em direção à região do visível. Tal efeito foi dependente da concentração de Ce, intensificando-se em função do aumento desta grandeza. A atividade fotocatalítica destes materiais foi testada na degradação de formaldeído. Para a fotoativação empregou-se radiação com comprimento de onda entre 300-600 nm, com um máximo de emissão em $365 \mathrm{~nm}$. A melhor resposta foi obtida para $\mathrm{O} \mathrm{TiO}_{2} / \mathrm{Ce} 0,1 \%$, com sua constante de pseudo primeira ordem para a degradação do formaldeído cerca de 4 vezes maior que a obtida para o $\mathrm{TiO}_{2}$ puro.

Além do deslocamento da energia de excitação, a incorporação de íons de cério em matriz de $\mathrm{TiO}_{2}$ também pode produzir uma diminuição do tamanho médio de partícula, já que a presença destes íons na superfície das partículas evita a aglomeração das mesmas durante o processo de síntese/calcinação ${ }^{65}$. Desta forma, em processos heterogêneos, quanto maior a área superficial do catalisador disponível, maior será a quantidade de $\mathrm{H}_{2} \mathrm{O}, \mathrm{O}_{2}$ e $\mathrm{OH}^{-}$ adsorvidos em sua superfície e, conseqüentemente, as reações de geração de radicais podem ser favorecidas. Ademais, o mecanismo direto de degradação dos compostos poluentes também pode ser favorecido com o aumento na superfície dos catalisadores ${ }^{65,66}$.

Além da dopagem, outra alternativa para a modificação das características dos catalisadores de óxidos metálicos é a utilização de óxidos mistos para produzir fotocatalisadores com características distintas dos óxidos de partida, as quais podem ser extremamente promissoras para a finalidade específica em que se deseja aplicar este semicondutor ${ }^{67,68}$.

A síntese e aplicação fotocatalítica de óxidos mistos de $\mathrm{TiO}_{2} \mathrm{e}$ $\mathrm{CeO}_{2}$ foi efetuada por Pavasupree e colaboradores ${ }^{67}$, que aplicaram o método de sol-gel assistido por surfactante para a síntese destes materiais. As proporções dos óxidos estudadas foram $90 \% \mathrm{TiO}_{2}$ $10 \% \mathrm{CeO}_{2}, 75 \% \mathrm{TiO}_{2}-25 \% \mathrm{CeO}_{2}, 50 \% \mathrm{TiO}_{2}-50 \% \mathrm{CeO}_{2}, 25 \% \mathrm{TiO}_{2}-$ $75 \% \mathrm{CeO}_{2}$. A característica mais relevante destes fotocatalisadores foi a grande capacidade de absorção de radiação em comprimentos de ondas maiores que os seus óxidos precursores. O efeito mais pronunciado foi observado para o óxido misto composto por $50 \% \mathrm{TiO}_{2}-50 \% \mathrm{CeO}_{2}$, que apresentou uma grande absorção para radiações com comprimentos de onda em torno de $460 \mathrm{~nm}$. A capacidade de absorção de radiação em comprimentos de ondas maiores dos catalisadores de óxidos mistos de $\mathrm{TiO}_{2}-\mathrm{CeO}_{2}$ foi correlacionada com sua atividade catalítica na oxidação do íon $\mathrm{I}^{-}$, com a realização do processo de fotocatálise heterogênea com radiações em comprimentos de ondas maiores que $400 \mathrm{~nm}$. A atividade catalítica do óxido composto por $50 \% \mathrm{TiO}_{2}-50 \% \mathrm{CeO}_{2}$ foi muito superior à obtida pelo processo fotocatalítico empregando apenas o $\mathrm{TiO}_{2}$ ou o $\mathrm{CeO}_{2}$, separadamente. A oxidação do íon $\mathrm{I}^{-}$em $\mathrm{I}_{3}^{-}$pelo $\mathrm{TiO}_{2}-\mathrm{CeO}_{2}$ foi cerca de 2 a 3 vezes maior que a do $\mathrm{CeO}_{2}$ puro, já o $\mathrm{TiO}_{2}$ não apresentou qualquer resposta catalítica, pois a radiação empregada não possibilitou sua fotoativação e, consequientemente, as reações responsáveis pela geração dos radicais não se processaram.

Óxidos mistos de $\mathrm{TiO}_{2}-\mathrm{CeO}_{2}$ também foram empregados na degradação de azul de metileno pelo processo de fotocatálise heterogênea empregando radiação na região do visível. Neste caso, também se evidenciou que os catalisadores de óxidos mistos possuem melhor atividade fotocatalítica que seus precursores puros ${ }^{68}$. Isto se deve, dentre outras propriedades, à formação de uma heterojunção nas interfaces do $\mathrm{TiO}_{2}$ e $\mathrm{CeO}_{2}$, através de ligações de Ti-O-Ce ${ }^{68}$. Nestas heterojunções é possível a excitação eletrônica em comprimentos de onda menos energéticos e os pares $\mathrm{h}^{+} / \mathrm{e}^{-}$podem ser transportados tanto para o $\mathrm{TiO}_{2}$ quanto para $\mathrm{O}^{\mathrm{CeO}}$ e prosseguir as reações de geração de radicais, Figura 4.

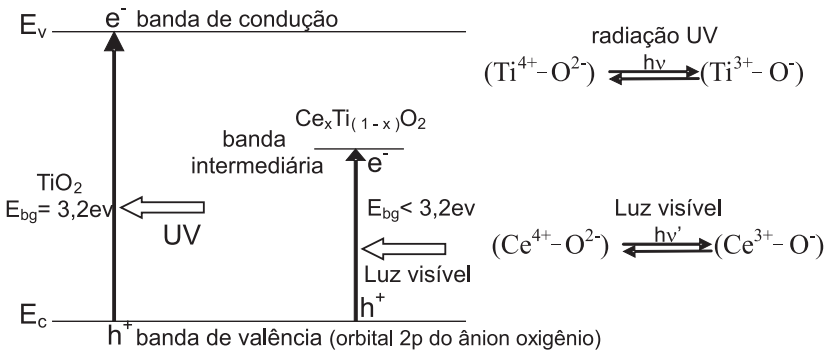

Figura 4. Esquema de fotoexcitação da banda intermediária formada na heterojunção entre óxidos de cério e titânio

Desta forma, tanto com a dopagem do $\mathrm{TiO}_{2}$ quanto nos óxidos mistos de $\mathrm{TiO}_{2}-\mathrm{CeO}_{2}$ estas regiões de interação, a heterojunção, possuem características, com relação ao "band-gap", distintas dos óxidos de titânio e cério individualmente. Assim, a formação de sítios oxidativos e redutivos ocorre em comprimentos de onda menos energéticos, próximo da região do espectro visível. As contribuições desta nova geração de catalisadores ao processo de fotocatálise heterogênea aumentam as possibilidades de emprego desta tecnologia de tratamento de poluentes orgânicos em grande escala.

\section{CONSIDERAÇÕES FINAIS}

De maneira geral, compostos de cério são empregados preferencialmente em sistemas heterogêneos. Assim, o desenvolvimento de materiais com melhores propriedades superficiais, tais como maior área superficial, porosidade e morfologia da partícula, contribuiu para ampliar as possibilidades do emprego do cério em sistemas em escala industrial.

Especificamente, sua aplicação em tecnologias ambientais, na degradação de compostos poluentes, apesar de se mostrar promissora, ainda é pouco explorada. No processo de fotocatálise heterogênea levando-se em consideração a menor energia de "band-gap" do $\mathrm{CeO}_{2}$ em comparação, por exemplo, ao $\mathrm{TiO}_{2}$, espera-se um menor potencial redox do par $\mathrm{h}^{+} / \mathrm{e}^{-}$gerado por sua excitação eletrônica, já que a diferença de energia entre a banda de valência e de condução é inversamente proporcional à atividade química dos transportadores de carga $\left(\mathrm{h}^{+} / \mathrm{e}^{-}\right)$. Desta forma, as lacunas $\left(\mathrm{h}^{+}\right)$geradas pelo cério são menos oxidantes que as geradas pelo $\mathrm{TiO}_{2}$, diminuindo a probabilidade de 
geração dos radicais e a oxidação de compostos orgânicos pelo mecanismo direto. A comparação do desempenho fotocatalítico de semicondutores deve ser efetuada de maneira controlada e criteriosa, levando em consideração as condições experimentais.

Além de suas propriedades individuais, a atuação dos íons de cério como dopantes na formação de óxidos mistos com outros semicondutores, como o $\mathrm{TiO}_{2}$, agrega aos fotocatalisadores características vantajosas, que são de grande importância para uma melhora na eficiência do processo de fotocatálise heterogênea.

Estas alternativas na modulação das partículas dos fotocatalisadores, juntamente com estratégias de dopagem e utilização de óxidos mistos, abrem uma nova perspectiva para os processos de fotocatálise heterogênea e oxidação por via úmida.

\section{AGRADECIMENTOS}

À FAPESP e ao CNPq pelo apoio financeiro.

\section{REFERÊNCIAS}

1. Greenwood, N. M.; Earnshaw, A.; Chemistry of the Elements, Pergamon Press: Great Britain, 1984.

2. Szabadvary, F. Em Handbook on the Physics and Chemistry of Rare Earths; Gschneidner Jr., K. A.; Eyring, L., eds.; Elsevier: Amsterdam, 1988, vol. 11.

3. Moeller, T.; The Chemistry of the Lanthanides, Pergamon Texts in Comprehensive Inorganic Chemistry; Pergamon Press: New York, 1975, vol. 26.

4. Puche, R. S.; Caro, P.; Rare Earths - Cursos de Verano de El Escorial, Editorial Complutense: Madrid, 1998.

5. Kilbourn, B. T.; A Lanthanide Lanthology, Molycorp, Inc., White Plains: New York, 1993.

6. Martins, T. S.; Isolani, P. C.; Quim. Nova 2005, 28, 111.

7. Carrijo, R. M. C.; Romero, J. R.; Quim. Nova 2000, 23, 331.

8. Vicentini, G.; Zinner, L. B.; Carvalho, L. R. F.; Folheto - Produção e Aplicações das Terras Raras, São Paulo, 1980.

9. Abrão, A.; Química e Tecnologia das Terras-Raras, CETEM/CNPq: Rio de Janeiro 1994.

10. Smektala, F.; Melscoest, I.; Fonteneau, G.; Lucas, J.; J. Non-Cryst. Solids 1998, 239, 156.

11. Paul, A.; Phys. Chem. Glasses 1976, 17, 7.

12. Fang, B.; Iwasa, S.; Wei, Y.; Arai, T.; Kumagai, M.; Electrochim. Acta 2002, 47, 3971.

13. Kostova, I.; Manolov, I.; Momekov, G.; Tzanova, T.; Konstantinov, S.; Karaivanova, M.; Eur. J. Med. Chem. 2005, 40, 1246.

14. Garner, J. P.; Heppell, P. S. J.; Burns 2005, 31, 539.

15. Wang, Y. H.; Zhang, J. C.; Fuel 2005, 84,1926.

16. Rey, J. F. Q.; Muccillo, E. N. S.; Cerâmica 2002, 48, 157

17. de Florio, D. Z.; Fonseca, F. C.; Muccillo, E. N. S.; Muccillo, R.; Cerâmica 2004, 50, 275

18. Dresselhaus, M. S.; Thomas, I. L.; Nature 2001, 414, 332.

19. Lokurlu, A.; Grube, T.; Höhlein, B.; Stolten, D.; Int. J. Hydrogen Energy 2003, 28, 703 .

20. Tang, L. C.; Yang, T. G.; J. Chem. Process 1995, 1, 18.

21. Trovarrell, A.: Catalytic Properties of Ceria and CeOl-Containing Materials, Marcel Dekker, Inc: Undine, 1996.

22. Trovarelli, A.; Boaro, M.; Rocchini, E.; Leitenburg, C.; Dolcetti, G.; J. Alloys Compd. 2001, 323, 584.

23. Coronado, J. M.; Maira, A. J.; Martínez-Arias, A.; Conesa, J. C.; Soria, J.; J. Photochem. Photobiol., A 2002, 150, 213.

24. Gutiérrez-Ortiz, J. I.; de Rivas, B.; López-Fonseca, R.; González-Velasco, J. R.; Appl. Catal., B 2006, 65, 191.

25. Lai, S. Y.; Qiu, Y. F.; Wang, S. J.; J. Catal. 2006, 237, 303.
26. Rangel, M. C.; Carvalho M. F. A.; Quim. Nova 2003, 26, 265.

27. Leitenburg, D. G. C.; Dolcetti, G.; Trovarelli, A.; J. Alloys Compd. 2006, 408-412, 1136.

28. Aneggi, E.; Boaro, M.; Leitenburg, C.; Dolcetti, G.; Trovarelli, A.; J. Alloys Compd. 2006, 408-412, 1096.

29. Shinjoh, H.; J. Alloys Compd. 2006, 408-412, 1061

30. Larachi, F.; Top. Catal. 2005, 33, 109.

31. Gorte, R. J.; Zhao, S.; Catal. Today 2005, 104, 18.

32. Hilaire, S.; Sharma, S.; Gorte, R. J.; Vohs, J. M.; Jen, H.-W.; Catal. Lett. 2000, 70, 131

33. Matsumoto, S.; Catal. Today 2004, 90, 183.

34. McCarty, J. G.; Nature 2000, 403, 35.

35. Sugiura, M.; Catal. Surv. Asia 2003, 7, 77.

36. Trovarelli, A.; Leitenburg, C.; Boaro, M.; Dolcetti, G.; Catal. Today 1999, $50,353$.

37. Silva, A. M. T.; Castelo-Branco, I. M.; Quinta-Ferreira, R. M.; Levec, J.; Chem. Eng. Sci. 2003, 58, 963.

38. Bueno-López, A.; Krishna, K.; Makkee, M.; Moulijn, J. A.; J. Catal. 2005 , 230, 237.

39. Silva, A. M. T.; Marques, R. R. N.; Quinta-Ferreira, R. M.; Appl. Catal., B 2004, 47, 269.

40. Liu, R.; Li. D. Y.; J. Mater. Sci. 2000, 35, 633.

41. Idriss, H.; Platinum Metals Rev. 2004, 48, 105.

42. Di Monte, R.; Kaspar, J.; Catal. Today 2005, 100, 27.

43. Colussi, S.; Leienburg, C.; Dolcetti, G.; Trovarelli, A.; J. Alloys Compd. 2004, 374, 287.

44. Corma, A.; Atienzar, P.; Garcia, H.; Chane-Ching, J. Y.; Nat. Mater. 2004 3, 394.

45. Chane-Ching, J. Y.; Airiau, M.; Sahibed-dine, A.; Daturi, M.; Brendlé, E.; Ozil, F.; Thorel, A.; Corma, A.; Langmuir 2005, 21, 1568.

46. Lin, S. S.; Chang, D. J.; Wang, C.H.; Chen, C. C.; Water Res. 2003, 37, 793.

47. Abecassis-Wolfovich, M.; Jothiramalingam, R.; Landau, M. V.; Herskowitz, M.; Viswanathan, B.; Varadarajan, T. K.; Appl. Catal., B 2005, 59, 91.

48. Oliviero, L.; Barbier, J. Jr.; Duprez, D.; Wahyu, H.; Ponton, J. W.; Metcalfe, I. S.; Mantzavinos, D.; Appl. Catal., B 2001, 35, 1 .

49. Hosokawa, S.; Kanai, H.; Utani, K.; Taniguchi, Y.; Saito, Y.; Imamura, S.; Appl. Catal., B 2003, 45, 181.

50. Chang, D. J.; Chen, I. P.; Chen, M. T.; Lin, S. S.; Chemosphere 2003, 52, 943.

51. Lin, S. S.; Chen, C. L.; Chang, D. J.; Chen, C. C.; Water Res. 2002, 36 , 3009.

52. Fox, M. A.; Dulay, M. T.; Chem. Rev. 1993, 93, 341.

53. Hoffmann, M. R.; Martin, S. T.; Choi, W.; Bahnemann, D. W.; Chem. Rev. 1995, 95, 69.

54. Kamat, P. V.; Chem. Rev. 1993, 93, 267.

55. Legrini, O.; Oliveros, E.; Braun, A. M.; Chem. Rev. 1993, 93, 671.

56. Zhang, Y.; Xu, H.; Xu, Y.; Zhang, H.; Wang, Y.; J. Photochem. Photobiol., A 2005, 170, 279.

57. Zhang, Y.; Zhang, H.; Xu, Y.; Wang, Y.; J. Solid State Chem. 2004, 177, 3490 .

58. Xie, Y.; Yuan, C.; Li, X.; Mater. Sci. Eng., B 2005, 117, 325.

59. Xu, W. A.; Gao, Y.; Liu, H. Q.; J. Catal. 2002, 207, 151.

60. Cho, Y.; Kyung, H.; Choi, W.; Appl. Catal., B 2004, 52, 23.

61. Coronado, J. M.; Maira, A. J.; Martínez-Arias, A.; Conesa, J. C.; Soria, J.; J. Photochem. Photobiol., A 2002, 150, 213.

62. Hernández-Alonso, M. D.; Hungrla, A. B.; Martínez-Arias, A.; FernándezGarcia, M.; Coronado, J. M.; Conesa, J. C.; Soria, J.; Appl. Catal., B 2004, $50,167$.

63. Oppenlander, T.; Photochemical purification of water and air, Wiley-VCH: Weinheim, 2003.

64. Xu, Y.; Chen, H.; Zeng, Z.; Lei, B.; Appl. Surf. Sci., 2006, 252, 8565.

65. Henderson, M. A.; Surf. Sci. Rep. 2002, 46, 1.

66. Shapovalov, V.; Stefanovich, E. V.; Truong, T. N.; Surf. Sci. 2002, 498, L103.

67. Pavasupree, S.; Suzuki, Y.; Pivsa-Art, S.; Yoshikawa, S.; J. Solid State Chem. 2005, 178, 128.

68. Liu, B.; Zhao, X.; Zhang, N.; Zhao, Q.; He, X.; Feng, J.; Surf. Sci. 2005, 595, 203. 